Erratum

\title{
An Involvement of Dopamine in Higher Order Choice Mechanisms in the Monkey
}

\author{
R. M. Ridley, T. A. J. Haystead, H. F. Baker
}

Psychopharmacology 72: 173-177 (1981)

The first paragraph of the Discussion (page 175) should read:

Since all animals performed almost $100 \%$ correctly on the simple discrimination tasks both before and after choice strategy trials, amphetamine did not seem to affect ability to perform the response, and did not grossly disrupt either perceptual mechanisms required to distinguish small objects or food-directed motivation.
The publisher apologizes for discrepancies in the originally published version, the result of copy-editing, production, and printing errors, some of which were made after the proofs had already been seen by the authors. The publisher acknowledges that the resulting errors are not the responsibility of the authors. 